\title{
Dispersion corrections to the forward Rayleigh scattering amplitudes around the L-shell of tantalum and lead
}

\section{S. B. Appaji Gowda and T. K. Umesh*}

Department of Studies in Physics, Manasagangothri, University of Mysore, Mysore-570006, India

Received 19 September 2005; Revised 27 April 2006; Accepted 8 May 2006

\begin{abstract}
Dispersion corrections to the forward Rayleigh scattering amplitudes of tantalum and lead in the photon energy range 6.4-24.14 keV were determined by a numerical evaluation of the dispersion integral that relates them through the optical theorem to the photoelectric cross-sections. The photoelectric cross-sections were extracted by subtracting the coherent and incoherent scattering contribution from the measured total attenuation cross-section, using a high-resolution, high-purity germanium detector in a narrow-beam good geometry setup. The real part of the dispersion correction to which the relativistic corrections calculated by Kissel and Pratt (S-matrix approach) or Creagh and McAuley (multipole corrections) have been included are in better agreement with the available theoretical values than those values to which the relativistic corrections calculated by Cromer and Liberman (dipole corrections) are added. Copyright ( 2006 John Wiley \& Sons, Ltd.
\end{abstract}

\section{INTRODUCTION}

It is known from dispersion theory that there exists a relation between the real and imaginary parts $f^{\prime}$ and $f^{\prime \prime}$ of the forward Rayleigh scattering amplitude (also called anomalous scattering factors) and photoelectric cross-section of the element. These relations shown in Eqns (1) and (2) are written based on the optical theorem: ${ }^{1}$

$$
f^{\prime \prime}(E)=\frac{1}{2 h c r_{0}} E \tau
$$

where $h c=1239.84 \mathrm{MeV} \mathrm{fm}, r_{0}=2.8179403 \times 10^{-13} \mathrm{~cm}$ is the classical electron radius and $\tau$ is the photoelectric crosssection of the element ${ }^{2}$ at the energy of interest $E$. The total cross-section $\sigma_{\text {tot }}-$ is given by $\sigma_{\text {tot }}=\tau+\sigma_{\mathrm{BBT}}-\sigma_{\mathrm{BPP}}$, where $\tau$, $\sigma_{\mathrm{BBT}}$ and $\sigma_{\mathrm{BPP}}$ are the photo-effect, photoexcitation and bound pair production cross-sections, respectively. For energies sufficiently away from absorption edge of a particular element, $\sigma_{\mathrm{BBT}}$ and $\sigma_{\mathrm{BPP}}$ are expected to be insignificant for $Z>10$, below the pair production threshold., In the energy region of current interest, if we neglect the spin flip process, the $f^{\prime}$ and $f^{\prime \prime}$ are connected by the modified Kramer's-Kronig transformation, given by

$$
f_{\mathrm{R}}^{\prime}(E)=f^{\prime}(\infty)-\left(\frac{2}{\pi}\right) P \int_{0}^{\infty} \frac{E^{\prime} f^{\prime \prime}\left(E^{\prime}\right)}{\left(E^{2}-E^{\prime 2}\right)} \mathrm{d} E^{\prime}
$$

where $P$ is the Cauchy principal value of the dispersion integral. The second term on the right-hand side represents $f_{\mathrm{NR}}^{\prime}(E)$. It is worth noting that the $f_{\mathrm{NR}}^{\prime}(E)$ values are the non-relativistic values. These values are found to be at

*Correspondence to: T. K. Umesh, Department of Studies in Physics, Manasagangothri, University of Mysore, Mysore-570006, India. E-mail: tku@physics.uni-mysore.ac.in variance with the theoretical values by a factor $f^{\prime}(\infty)$ which is called the high-energy limit or the relativistic correction. It is negative and is generally independent of the energy. $f^{\prime}(\infty)$ values have been provided by Cromer and Liberman (CL), Creagh and McAuley (CM) and Kissel and Pratt (KP). Among these, the CL corrections are obtained in the dipole approximation; the $\mathrm{CM}$ corrections are based on the theoretical treatment that includes multipole approximation in addition to retardation effects. ${ }^{5}$ The KP correction calculations are based on the S-matrix approach. The $\mathrm{CM}$ and $\mathrm{KP}$ corrections are considered to be by far the most accurate. Hence it is possible to determine the dispersion corrections $f^{\prime}$ and $f^{\prime \prime}$ from a set of photoelectric cross-sections using Eqns 1 and 2.

Ever since the first theoretical prediction of $x$-ray dispersion by Waller, ${ }^{6}$ there have been a number of attempts to evaluate the dispersion corrections $f^{\prime}$ and $f^{\prime \prime}$. Based on Waller's ${ }^{6}$ theory, for a hydrogen-like atom in a nonrelativistic approach, Cromer and Liberman ${ }^{7}$ have given extensive tabulations of $f^{\prime}$ and $f^{\prime \prime}$ over a range of wavelengths commonly used by crystallographers. Creagh and McAuley ${ }^{8}$ have given tabulations of $f^{\prime}$ and $f^{\prime \prime}$. Kissel and Pratt ${ }^{9}$ have tabulated the values of the high-energy limit, based on Smatrix calculations, to be added to $f^{\prime}$ and also given the values of correction to be added to the Cromer-Liberman $f^{\prime}$ values. Chantler ${ }^{10}$ has provided an extensive tabulation of theoretical form factor, attenuation and scattering data for elements of $Z=1-92$ from $E=1-10 \mathrm{eV}$ to $0.4-1 \mathrm{MeV}$. Cullen et al. ${ }^{11}$ have provided a photon data library (EPDL97). This library includes photon interaction data for all elements with atomic number range 1-100 and energy range $1 \mathrm{eV}-100 \mathrm{GeV} ; f^{\prime}$ and $f^{\prime}$ values are also provided. The relativistic correction term used was that calculated by Kissel and Pratt. ${ }^{9}$ 
On the experimental side, two types of techniques have mainly been used to determine $f^{\prime}$ and $f^{\prime \prime}$. These are the direct method and the attenuation coefficient method. The direct method is based on (i) the measurement of the refractive index of the specimen of interest, (ii) the determination of the intensities of Bragg reflections ${ }^{12}$ and (iii) x-ray interferometery ${ }^{13}$ from which $f^{\prime}$ and $f^{\prime \prime}$ values are calculated. The attenuation coefficient method can also be used to obtain the dispersion corrections $f^{\prime}$ and $f^{\prime \prime}$. Sandiago et al., ${ }^{14}$ Umesh et al. ${ }^{5}$ and Appaji Gowda et al. ${ }^{15}$ have evaluated dispersion corrections around the $\mathrm{K}$-shell for the elements $\mathrm{Cu}, \mathrm{Ag}$, Zr, Sn, La, Ce, Pr, Nd, Sm, Gd, Dy, Ho and $\mathrm{Er}$, in the energy range $5-84 \mathrm{keV}$ using the attenuation coefficient method. Drier et al. ${ }^{16}$ have reported results for $\mathrm{Ni}$, $\mathrm{Cu}, \mathrm{Zn}$ and $\mathrm{Zr}$ around the K-edge and for Ta, W, Pt and $\mathrm{Au}$ around the respective L-edges.

In this method, $f^{\prime}$ and $f^{\prime \prime}$ are evaluated by first measuring the total attenuation cross-sections of elements over a wide range in the low-energy $x$-ray and $\gamma$-ray region (below $100 \mathrm{keV}$ ). From these data, atomic photoelectric crosssections are derived by subtracting the relatively insignificant scattering cross-sections. These atomic photoelectric crosssections are further used to evaluate $f^{\prime}$ and $f^{\prime \prime}$. The evaluation of $f^{\prime}$ from $f^{\prime \prime}$ involves a numerical computation of the dispersion integral (2). Thus, the $f_{\mathrm{NR}}^{\prime}$ values calculated using the photoelectric cross-sections can be used to determine which one of these $f^{\prime}(\infty)$ values renders a good comparison of $f_{\mathrm{R}}^{\prime}(E)$ values with the theoretical data.

In this paper, we report $f_{\mathrm{R}}^{\prime}(E)$ and $f^{\prime}$ values obtained for Ta and $\mathrm{Pb}$. The $f_{\mathrm{R}}^{\prime}(E)$ values are calculated by separately adding the relativistic corrections $f^{\prime}(\infty)$ to the $f_{\mathrm{NR}}^{\prime}$ values. The resulting values are compared with the available theoretical data of Chantler ${ }^{10}$ and Cullen et al. ${ }^{11}$

\section{EXPERIMENTAL}

We measured the total attenuation cross-sections of tantalum and lead in the energy range of interest in a narrow beam, good geometry setup. The detector was a high-resolution hyper-pure germanium detector. The details regarding the experimental setup, method of data acquisition and error analysis along with the calculation of the total attenuation cross-sections are given in an earlier paper. ${ }^{17}$

\section{RESULTS AND DISCUSSION}

From the measured total attenuation cross-section values of the tantalum and lead shown in Table 1, the photoelectric cross-sections $\tau$ were derived by subtracting the theoretical scattering contribution based on the XCOM values of Berger and Hubbell. ${ }^{18}$ Using the corresponding values of the photoelectric cross-section so obtained for energies $E$ both above and below the L-shell, the imaginary part $f^{\prime}(E)$ of the dispersion correction was calculated using Eqn (1) at each energy $E$. These values are given in Table 2.

Furthermore, the $f^{\prime}(E)$ values were used to calculate the $f_{\mathrm{NR}}^{\prime}(E)$ values by a numerical evaluation of the dispersion integral. For this, the lower limit of integration was chosen to
Table 1. Photoelectric cross-sections in barn/atom (errors are to the extent of $3 \%{ }^{17}$ )

\begin{tabular}{lcccrr}
\hline & \multicolumn{2}{c}{ Tantalum } & & \multicolumn{2}{c}{ Lead } \\
\cline { 2 - 3 } \cline { 6 - 6 } Energy $(\mathrm{keV})$ & $\begin{array}{l}\text { Present } \\
\text { values }\end{array}$ & $\begin{array}{c}\text { XCOM } \\
\text { values }\end{array}$ & & $\begin{array}{c}\text { Present } \\
\text { values }\end{array}$ & $\begin{array}{r}\text { XCOM } \\
\text { values }\end{array}$ \\
\hline 6.4 & 81900 & 84430 & & 130368 & 134400 \\
8.014 & 44650 & 47410 & 75600 & 76350 \\
10.53 & 58045 & 61100 & 36656 & 37790 \\
14.4 & 43682 & 43720 & & 40750 & 41580 \\
24.14 & 10692 & 11100 & 17341 & 17600 \\
\hline
\end{tabular}

Table 2. Imaginary part of anomalous scattering factor $\left(f^{\prime}\right)$ in e/atom

\begin{tabular}{lcccccccc}
\hline & \multicolumn{3}{c}{ Tantalum } & & \multicolumn{3}{c}{ Lead } \\
\cline { 2 - 4 } \cline { 7 - 8 } $\begin{array}{l}\text { Energy } \\
(\mathrm{keV})\end{array}$ & $\begin{array}{c}\text { Present } \\
\text { values }\end{array}$ & $\begin{array}{c}\text { Chantler } \\
\text { values }\end{array}$ & $\begin{array}{c}\text { Cullen } \\
\text { values }\end{array}$ & & $\begin{array}{c}\text { Present } \\
\text { values }\end{array}$ & $\begin{array}{c}\text { Chantler } \\
\text { values }\end{array}$ & $\begin{array}{c}\text { Cullen } \\
\text { values }\end{array}$ \\
\hline 6.4 & 7.50 & 7.35 & 7.77 & & 11.94 & 11.84 & 12.39 \\
8.041 & 5.12 & 5.26 & 5.47 & & 8.70 & 8.43 & 8.77 \\
10.53 & 8.75 & 9.14 & 9.26 & & 5.53 & 5.50 & 5.69 \\
14.4 & 9.00 & 8.83 & 8.99 & & 8.40 & 8.47 & 8.61 \\
24.14 & 3.69 & 3.79 & 3.83 & & 5.99 & 6.01 & 6.09 \\
\hline
\end{tabular}

be the $\mathrm{M}_{\mathrm{V}}$ absorption edge and the upper limit was $1332 \mathrm{keV}$. Below $6.4 \mathrm{keV}$ up to the $\mathrm{M}_{\mathrm{V}}$ edge, photoelectric cross-section data based on XCOM were used since the measurements were made only at and above $6.4 \mathrm{keV}$ in this work. From 6.4 up to $24.14 \mathrm{keV}$ the values given in Table-1 were used. Beyond $24.14 \mathrm{keV}$, measured values reported earlier were used. ${ }^{19-22}$ To evaluate the integral numerically, the energy region was divided into a large number of small intervals. Within each interval $\left(E_{i}, E_{i+1}\right)$, the energy dependence of $f_{i}^{\prime}(E)$ was determined by a linear function:

$$
f_{i}^{\prime}(\mathrm{E})=\left(a_{i}+b_{i} E\right)
$$

In this interval, the dispersion integral assumes the form

$$
I_{i, i+1}\left(E_{s}\right)=\frac{2}{\pi} P \int_{E_{i}}^{E_{i+1}} \frac{E^{\prime} f^{\prime \prime}\left(E^{\prime}\right)}{E_{s}^{2}-E^{\prime 2}} \mathrm{~d} E^{\prime}
$$

Mathematically, the above expression is equivalent to

$$
\begin{aligned}
I_{i, i+1}\left(E_{s}\right)= & -\frac{2}{\pi}\left[\frac{a_{i}}{2} \ln \left|\frac{E_{s}^{2}-E_{i+1}^{2}}{E_{s}^{2}-E_{i}^{2}}\right|\right. \\
& \left.+b_{i}\left(E_{i+1}-E_{i}-\frac{E_{s}}{2} \ln \left|\frac{\left(E_{i+1}+E_{s}\right)}{\left(E_{i+1}-E_{s}\right)} \frac{\left(E_{i}-E_{s}\right)}{\left(E_{i}+E_{s}\right)}\right|\right)\right](5)
\end{aligned}
$$

Using the coefficients $a_{i}$ and $b_{i}$, the dispersion integral was calculated for each interval. The integrals $I_{s-1, s}$ and $I_{s, s+1}$ which are not defined are replaced at once by the integral $I_{s-1, s+1}$. Here, $E_{s-1}$ and $E_{s+1}$ are energies very close to the energy of interest $E_{s}$. The final value of the dispersion integral is obtained simply by adding all ${ }_{I i, i+1}$ values. From these $f_{\mathrm{NR}}^{\prime}$ values, the relativistic correction $f^{\prime}(\infty)$ suggested by different authors, Cromer and Liberman ${ }^{7}$ (dipole correction), 
Table 3. Relativistic correction $f^{\prime}(\infty)$ values

\begin{tabular}{lccc}
\hline CM (multipole \\
Element & 1992 & $\begin{array}{c}\text { KP (S-matrix } \\
\text { correction) } \\
1990\end{array}$ & $\begin{array}{c}\text { CL (dipole } \\
\text { correction) } \\
1970\end{array}$ \\
\hline Tantalum & -0.834 & -0.745 & -1.385 \\
Lead & -1.116 & -0.986 & -1.856 \\
\hline
\end{tabular}

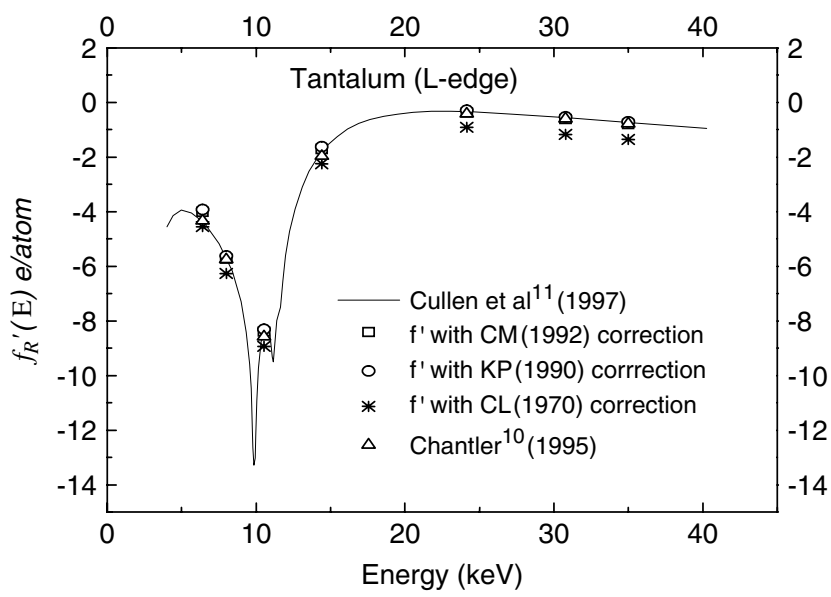

Figure 1. $f_{\mathrm{R}}^{\prime}(E)$ as a function of energy around the L-edge for tantalum.

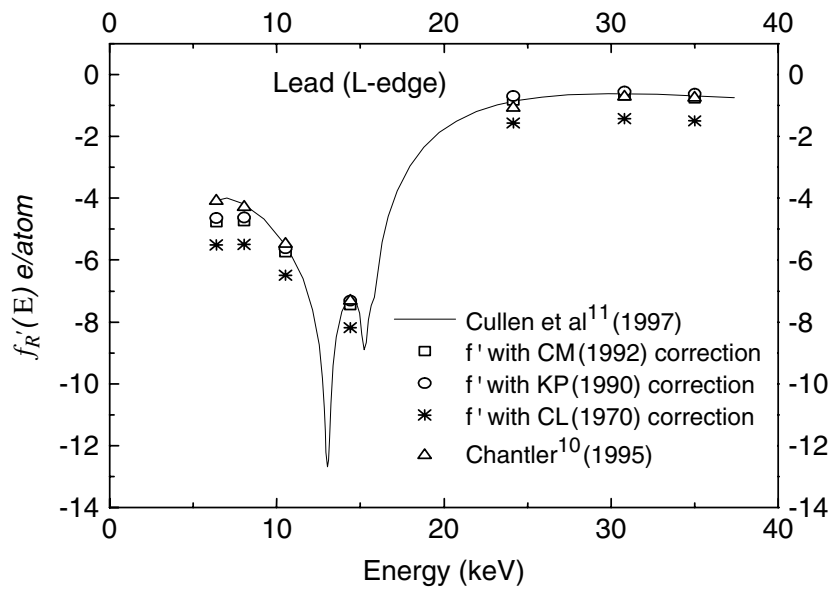

Figure 2. $f_{\mathrm{R}}^{\prime}(E)$ as a function of energy around the L-edge for lead.

Creagh and McAuley ${ }^{8}$ (multipole correction) and Kissel and Pratt $^{9}$ (S-matrix correction), for tantalum and lead were separately added and the values of $f^{\prime}$ were calculated for the elements. The values of $f^{\prime}(\infty)$ employed are shown in Table 3.

The $f_{\mathrm{R}}^{\prime}$ values obtained in the case of Ta and $\mathrm{Pb}$ are shown in Figs 1 and 2 along with the theoretical data of Cullen et al. ${ }^{11}$ $(\mathrm{KP})$ and $\mathrm{Chantler}^{10}(\mathrm{CM})$. It can be seen that the values of
$f_{R}^{\prime}(E)$ in which CM or KP correction is included follow the trend suggested by theory.

\section{CONCLUSIONS}

Based on this study, reasonable values of the dispersion corrections $f^{\prime}$ and $f^{\prime \prime}$ could be obtained from a fairly accurate total attenuation cross-section data set measured in a narrow beam, good geometry setup by employing a high-resolution detector to detect photons emitted by radioisotopes. The present study points to the fact that within the range of experimental errors, the $f_{R}^{\prime}(E)$ values in which the $f^{\prime}(\infty)$ values given by Creagh and $\mathrm{McAuley}^{8}$ or Kissel and Pratt $^{9}$ have been included are in better agreement with the theoretical values of Chantler ${ }^{10}$ and Cullen et al. ${ }^{11}$ than those values to which the relativistic corrections calculated by Cromer and Liberman ${ }^{7}$ (dipole corrections)are added. This conclusion also follows from Chantler's paper. ${ }^{10}$

\section{REFERENCES}

1. Nussenzveig HM. Causality and Dispersion Relations. Academic Press: New York, 1972; 183.

2. Mohr PJ, Taylor BN. Rev. Mod. Phys. 2005; 77: 1.

3. Wang MS. Phys. Rev.A 1986; 34: 636.

4. Wang MS, Pratt RH. Phys. Rev. A 1983; 28: 3115.

5. Umesh TK, Sandiago, Ramakrishna Gowda. Radiat. Phys. Chem. 1998; 51: 373

6. Waller I. Z. Phys. 1928; 51: 213.

7. Cromer T, Liberman D. J. Chem. Phys. 1970; 53: 1891.

8. Creagh DC, McAuley WJ. Int. Tables Crystallogr. C 1992; 206.

9. Kissel L, Pratt RH. Acta Crystallogr., Sect. A 1990; 46: 170.

10. Chantler CT. J. Phys. Chem. Ref. Data 1995; $24: 71$.

11. Cullen DE, Dermott E, Hubbell J, Kissel L. The Evaluated Photon Data Library, 97 version. Lawrence Livermore National Laboratory: Livermore, CA, 1997.

12. Fukamachi $\mathrm{T}$, Hosoya $\mathrm{S}$, Kawamura $\mathrm{T}$, Hastings J. J. Appl. Crystallogr. 1977; 10: 321.

13. Bonse U, Hartmann-Lotsch I, Lotsch H. Nucl. Instrum. Methods 1983; 208: 603.

14. Sandiago, Umesh TK, Ramakrishna Gowda. Pramana-J. Phys. 1997; 48: 1077.

15. Appaji Gowda SB, Mallikarjuna ML, Gowda R, Umesh TK. Pramana-J. Phys. 2003; 61: 539.

16. Drier P, Rabe P, Malzfeldt W, Niemann W. J. Phys. C 1984; 17: 3123.

17. Mallikarjuna ML, Appaji Gowda SB, Gowda R, Umesh TK. Radiat. Phys. Chem. 2002; 65: 217.

18. Berger MJ, Hubbell JH. XCOM, Photon Cross-sections on Personal Computer. National Bureau of Standards Internal Report No. 87. National Bureau of Standards: Washington, DC, 1987; 3597.

19. Umesh TK, Gowda R, Sanjeevaiah B. Phys. Rev. A 1982; 25: 1986.

20. Umesh TK, Ranganathaiah C, Sanjeevaiah B. Phys. Rev. A 1984; 29: 387.

21. Umesh TK, Ranganathaiah C, Sanjeevaiah B. Phys. Rev. A 1985; 32: 959.

22. Umesh TK, Anasuya SJ, Shylajakumari J, Channegowda, Gopinathan Nair KP, Ramakrishna Gowda. Phys. Rev. A 1992; 45: 2101. 